\title{
Bianchi Type II, VIII, and IX Perfect Fluid Dark Energy Cosmological Models in Saez-Ballester and General Theory of Gravitation
}

\author{
V. U. M. Rao, K. V. S. Sireesha, and D. Neelima \\ Department of Applied Mathematics, Andhra University, Visakhapatnam, A.P. 530003, India \\ Correspondence should be addressed to V. U. M. Rao; umrao57@hotmail.com
}

Received 2 December 2012; Accepted 20 December 2012

Academic Editors: H. Bushouse, I. Goldman, M. Grewing, and V. Pierrard

Copyright (C) 2013 V. U. M. Rao et al. This is an open access article distributed under the Creative Commons Attribution License, which permits unrestricted use, distribution, and reproduction in any medium, provided the original work is properly cited.

The paper deals with spatially homogeneous anisotropic Bianchi type II, VIII , and IX dark energy cosmological models filled with perfect fluid in the framework of Saez-Ballester (1986) theory, and Einstein's general relativity. Assuming that the two sources interact minimally and therefore their energy momentum tensors are conserved separately, we have considered different cases and presented anisotropic as well as isotropic cosmological models. Some important physical and geometrical features of the models, thus obtained, have been discussed.

\section{Introduction}

Saez and Ballester [1] formulated a scalar tensor theory of gravitation in which the metric is coupled with a dimensionless scalar field in a simple manner. This coupling gives a satisfactory description of the weak fields. In spite of the dimensionless character of the scalar field an antigravity regime appears. This theory also suggests a possible way to solve missing matter problem in nonflat FRW cosmologies.

The field equations given by Saez-Ballester [1] for the combined scalar and tensor fields (using geometrized units with $c=1,8 \pi G=1$ ) are

$$
G_{i j}-\omega \phi^{r}\left(\phi_{, i} \phi_{, j}-\frac{1}{2} g_{i j} \phi_{, k} \phi^{\prime k}\right)=-T_{i j}
$$

and the scalar field $\phi$ satisfies the equation

$$
2 \phi^{r} \phi_{, i}^{i}+r \phi^{r-1} \phi_{, k} \phi^{\prime k}=0,
$$

where $G_{i j}=R_{i j}-(1 / 2) R g_{i j}$ is an Einstein tensor, $R$ is the scalar curvature, $\omega$ and $r$ are constants, and $T_{i j}$ is the stress energy tensor of the matter.

The energy conservation equation is

$$
T_{, j}^{i j}=0 .
$$

The study of cosmological models in the framework of scalar tensor theories has been the active area of research for the last few decades. In particular, Rao et al. [2] and Rao et al. $[3,4]$ are some of the authors who have investigated several aspects of the cosmological models in Saez-Ballester [1] scalar tensor theory. Naidu et al. [5-8] have discussed various aspects of Bianchi space times in Saez-Ballester [1] scalar tensor theory.

Recent observations of type Ia supernovae (SN Ia) [9-13], galaxy redshift surveys [14], cosmic microwave background radiation (CMBR) data $[15,16]$, and large scale structure [17] strongly suggest that the observable universe is undergoing an accelerated expansion. Observations also suggest that there had been a transition of the universe from the earlier deceleration phase to the recent acceleration phase [18]. The cause of this sudden transition and the source of the accelerated expansion are still unknown. Measurements of CMBR anisotropies, most recently by the WMAP satellite, indicate that the universe is very close to flat. For a flat universe, its energy density must be equal to a certain critical density, which demands a huge contribution from some unknown energy stuff. Thus, the observational effects like the cosmic acceleration, sudden transition, flatness of universe, and many more need explanation. It is generally believed that some sort of "dark energy" (DE) is pervading the whole 
universe. It is a hypothetical form of energy that permeates all of space and tends to increase the rate of expansion of the universe [19]. The most recent WMAP observations indicate that $\mathrm{DE}$ accounts for $72 \%$ of the total mass energy of the universe [20]. However, the nature of DE is still a mystery.

Many cosmologists believe that the simplest candidate for the DE is the cosmological constant $(\Lambda)$ or vacuum energy since it fits the observational data well. During the cosmological evolution, the $\Lambda$ term has the constant energy density and pressure $p^{(\mathrm{de})}=-\rho^{(\mathrm{de})}$, where the superscript (de) stands for DE. However, one has the reason to dislike the cosmological constant since it always suffers from the theoretical problems such as the "fine-tuning" and "cosmic coincidence" puzzles [21]. That is why the different forms of dynamically changing DE with an effective equation of state $(\mathrm{EoS}), \omega^{(\mathrm{de})}=$ $p^{(\mathrm{de})} / \rho^{(\mathrm{de})}<-1 / 3$, have been proposed in the literature. Other possible forms of DE include quintessence $\left(\omega^{(\mathrm{de})}>-1\right)$ [22], phantom $\left(\omega^{(\mathrm{de})}<-1\right)$ [23], and so forth. While the possibility $\omega^{(\mathrm{de})} \ll-1$ is ruled out by current cosmological data from SN Ia (Supernovae Legacy Survey, Gold sample of Hubble Space Telescope) [13, 24], CMBR (WMAP, BOOMERANG) $[25,26]$ and large scale structure (Sloan Digital Sky Survey) [27] data, the dynamically evolving DE crossing the phantom divide line (PDL) $\left(\omega^{(\mathrm{de})}=-1\right)$ is mildly favored. SN Ia data combined with CMBR anisotropy and galaxy clustering statistics suggest that $-1.33<\omega^{(\mathrm{de})}<-0.79$ (see [28]).

Most of the models with constant DP have been studied by considering perfect fluid or ordinary matter in the universe. But the ordinary matter is not enough to describe the dynamics of an accelerating universe as mentioned earlier. This motivates the researchers to consider the models of the universe filled with some exotic type of matter such as the DE along with the usual perfect fluid. Recently, some dark energy models with constant DP have been investigated by Kumar and Singh [29], Akarsu and Kilinc [30-32], Yadav et al. [33], A. K. Yadav and L. Yadav [34], Pradhan et al. [35], and Rao et al. [36, 37]. Reddy et al. [38] have discussed five dimensional dark energy models in a Saez-Ballester [1] scalar tensor theory.

In this paper, we will discuss minimally interacting perfect fluid and dark energy Bianchi type II, VIII, and IX space-times in a scalar tensor theory of gravitation proposed by Saez and Ballester [1] and general theory of gravitation.

\section{Metric and Field Equations}

We consider spatially homogeneous Bianchi type II, VIII, and IX metrics in the form

$$
d s^{2}=-d t^{2}+R^{2}\left[d \theta^{2}+f^{2}(\theta) d \phi^{2}\right]+S^{2}[d \varphi+h(\theta) d \phi]^{2}
$$

where $\theta, \phi$, and $\varphi$ are the Eulerian angles. Also $R$ and $S$ are functions of $t$ only.
It represents

$$
\begin{aligned}
& \text { Bianchi type II if } f(\theta)=1 \text { and } h(\theta)=\theta \\
& \text { Bianchi type VIII if } f(\theta)=\cosh \theta \text { and } h(\theta)=\sinh \theta \\
& \text { Bianchi type IX if } f(\theta)=\sin \theta \text { and } h(\theta)=\cos \theta
\end{aligned}
$$

The energy momentum tensor is given by

$$
T_{i}^{j}=T_{j}^{(m) i}+T_{j}^{(\mathrm{de}) i}
$$

where $T_{j}^{(m) i}$ and $T_{j}^{(\mathrm{de}) i}$ are the energy momentum tensors of ordinary matter and DE, respectively, and are given by

$$
\begin{aligned}
T_{j}^{(m) i} & =\operatorname{diag}\left[-\rho^{(m)}, p^{(m)}, p^{(m)}, p^{(m)}\right] \\
& =\operatorname{diag}\left[-1, w^{(m)}, w^{(m)}, w^{(m)}\right] \rho^{(m)}, \\
T_{j}^{(\mathrm{de}) i} & =\operatorname{diag}\left[-\rho^{(\mathrm{de})}, p^{(\mathrm{de})}, p^{(\mathrm{de})}, p^{(\mathrm{de})}\right] \\
& =\operatorname{diag}\left[-1, w^{(\mathrm{de})}, w^{(\mathrm{de})}, w^{(\mathrm{de})}\right] \rho^{(\mathrm{de})},
\end{aligned}
$$

where $\rho^{(m)}$ and $p^{(m)}$ are the energy density and pressure of the perfect fluid component or ordinary baryonic matter while $w^{(m)}=p^{(m)} / \rho^{(m)}$ is its EoS parameter. Similarly, $\rho^{(\mathrm{de})}$ and $p^{(\mathrm{de})}$ are the energy density and pressure of the DE component while $w^{(\mathrm{de})}=p^{(\mathrm{de})} / \rho^{(\mathrm{de})}$ is the corresponding EoS parameter.

Now with the help of (5) to (7), the field equation (1) for the metric (4) can be written as

$$
\begin{gathered}
\frac{\ddot{R}}{R}+\frac{\ddot{S}}{S}+\frac{\dot{R} \dot{S}}{R S}+\frac{S^{2}}{4 R^{4}}-\frac{\omega}{2} \phi^{r} \dot{\phi}^{2}=-w^{(m)} \rho^{(m)}-w^{(\mathrm{de})} \rho^{(\mathrm{de})}, \\
2 \frac{\ddot{R}}{R}+\frac{\dot{R}^{2}+\delta}{R^{2}}-\frac{3 S^{2}}{4 R^{4}}-\frac{\omega}{2} \phi^{r} \dot{\phi}^{2}=-w^{(m)} \rho^{(m)}-w^{(\mathrm{de})} \rho^{(\mathrm{de})}, \\
2 \frac{\dot{R} \dot{S}}{R S}+\frac{\dot{R}^{2}+\delta}{R^{2}}-\frac{S^{2}}{4 R^{4}}+\frac{\omega}{2} \phi^{r} \dot{\phi}^{2}=\rho^{(m)}+\rho^{(\mathrm{de})}, \\
\ddot{\phi}+\dot{\phi}\left(2 \frac{\dot{R}}{R}+\frac{\dot{S}}{S}\right)+\frac{r}{2 \phi} \dot{\phi}^{2}=0 .
\end{gathered}
$$

The conservation equation yields

$$
\dot{\rho}^{(m)}+3\left(1+w^{(m)}\right) \rho^{(m)} H+\dot{\rho}^{(\mathrm{de})}+3\left(1+w^{(\mathrm{de})}\right) \rho^{(\mathrm{de})} H=0,
$$

where $H$ is themean Hubble parameter.

\section{Solutions of the Field Equations}

In order to solve the field equations completely, we assume that the perfect fluid and DE components interact minimally. Therefore, the energy momentum tensors of the two sources may be conserved separately. 
The energy conservation equation $T_{; j}^{(m) i j}=0$ of the perfect fluid leads to

$$
\dot{\rho}^{(m)}+3\left(1+w^{(m)}\right) \rho^{(m)} H=0,
$$

whereas the energy conservation equation $T_{; j}^{(\mathrm{de}) i j}=0$ of the DE component yields

$$
\dot{\rho}^{(\mathrm{de})}+3\left(1+w^{(\mathrm{de})}\right) \rho^{(\mathrm{de})} H=0 .
$$

Following Akarsu and Kilinc [30], we assume that the EoS parameter of the perfect fluid is a constant, that is,

$$
w^{(m)}=\frac{p^{(m)}}{\rho^{(m)}}=\text { cons tan } t
$$

while $w^{(\mathrm{de})}$ has been allowed to be a function of time since the current cosmological data from SN Ia, CMB, and large scale structures mildly favor dynamically evolving $\mathrm{DE}$ crossing the phantom divide line (PDL).

Now, we assume that the shear scalar $(\sigma)$ in the models is proportional to expansion scalar $(\theta)$.

This condition leads to

$$
S=R^{n},
$$

where $R$ and $S$ are the metric potentials and $n$ is a positive constant.

Since we are looking for a model explaining an expanding universe with acceleration, we also assume that the anisotropic distribution of $\mathrm{DE}$ ensures the present accelerating universe. Thus (8) to (12) may be rewritten as

$$
\begin{gathered}
\frac{\ddot{R}}{R}+\frac{\ddot{S}}{S}+\frac{\dot{R} \dot{S}}{R S}+\frac{S^{2}}{4 R^{4}}-\frac{\omega}{2} \phi^{r} \dot{\phi}^{2} \\
=-w^{(m)} \rho^{(m)}-\left(w^{(\mathrm{de})}+\gamma\right) \rho^{(\mathrm{de})}, \\
2 \frac{\ddot{R}}{R}+\frac{\dot{R}^{2}+\delta}{R^{2}}-\frac{3 S^{2}}{4 R^{4}}-\frac{\omega}{2} \phi^{r} \dot{\phi}^{2} \\
=-w^{(m)} \rho^{(m)}-\left(w^{(\mathrm{de})}+\xi\right) \rho^{(\mathrm{de})}, \\
2 \frac{\dot{R} \dot{S}}{R S} \frac{\dot{R}^{2}+\delta}{R^{2}}-\frac{S^{2}}{4 R^{4}}+\frac{\omega}{2} \phi^{r} \dot{\phi}^{2}=\rho^{(m)}+\rho^{(\mathrm{de})}, \\
\ddot{\phi}+\dot{\phi}\left(2 \frac{\dot{R}}{R}+\frac{\dot{S}}{S}\right)+\frac{r}{2 \phi} \dot{\phi}^{2}=0, \\
\dot{\rho}^{(\mathrm{de})}+3\left(1+w^{(\mathrm{de})}\right) \rho^{(\mathrm{de})} H+\rho^{(\mathrm{de})}\left(2 \gamma H_{x}+\xi H_{z}\right)=0 .
\end{gathered}
$$

The third term of (21) arises due to the deviation from $w^{(\mathrm{de})}$ while the first two terms of (21) are deviation free part of $T_{j}^{(\mathrm{de}) i}$. According to (21), the behavior of $\rho^{(\mathrm{de})}$ is controlled by the deviation free part of EoS parameter of DE but deviation will affect $\rho^{(\mathrm{de})}$ indirectly, since as can be seen later, they affect the value of EoS parameter. But we are looking for physically viable models of the universe consistent with observations. Hence we constrained $\xi(t)$ and $\gamma(t)$ by assuming the special dynamics which are consistent with (21). The dynamics of skewness parameter on $x$-axis or $y$-axis $\gamma$ and $z$-axis $\xi$ are given by

$$
\begin{aligned}
& \gamma=\frac{-m H H_{z}}{\rho^{(\mathrm{de})}}, \\
& \xi=\frac{2 m H H_{x}}{\rho^{(\mathrm{de})}},
\end{aligned}
$$

where $m$ is the dimensionless constant that parameterizes the amplitude of the deviation from $w^{(\mathrm{de})}$ and can be given real values.

From (17) and (18), we get

$$
\frac{\ddot{R}}{R}+C_{1} \frac{\dot{R}^{2}}{R^{2}}-\frac{\delta}{(n-1) R^{2}}+\frac{R^{2 n-4}}{(n-1)}=0, \quad n \neq 1,
$$

where $C_{1}=\left(3\left(n^{2}-1\right)-m(n+2)^{2}\right) / 3(n-1)$.

3.1. Bianchi Type II $(\delta=0)$ Cosmological Model. If $\delta=0$, (24) can be written as

$$
\frac{\ddot{R}}{R}+C_{1} \frac{\dot{R}^{2}}{R^{2}}+\frac{R^{2 n-4}}{(n-1)}=0, \quad n \neq 1 .
$$

From (25), we get

$$
R=\left(k_{1} t+k_{2}\right)^{1 /(2-n)}, \quad n \neq 2,
$$

where $n^{2}\left(1+k_{1}^{2}\right)+n\left(\left(C_{1}-2\right) k_{1}^{2}-4\right)+\left(\left(1-C_{1}\right) k_{1}^{2}+4\right)=$ $0, k_{1} \neq 0$ and $k_{2}$ are arbitrary constants.

From (16) and (26), we get

$$
S=\left(k_{1} t+k_{2}\right)^{n /(2-n)},
$$

From (11), (16), and (26) we get

$$
\begin{array}{r}
\phi^{(r+2) / 2}=\left[\frac{r+2}{2}\left(\frac{(n-2) k_{3}}{2 n}\left(k_{1} t+k_{2}\right)^{2 n /(n-2)}+k_{4}\right)\right], \\
r \neq-2, \quad n \neq 0,
\end{array}
$$

where $k_{3}$ and $k_{4}$ are integrating constants.

From (13), we get the energy density of the perfect fluid

$$
\rho^{(m)}=\rho_{0}\left(k_{1} t+k_{2}\right)^{-\left(1+w^{(m)}\right)(n+2) /(2-n)},
$$

where $\rho_{0}$ is a constant of integration.

From (19) and (26)-(29), we get the dark energy density

$$
\begin{aligned}
\rho^{(\mathrm{de})}= & \frac{\left[n\left(8 k_{1}^{2}+4\right)+\left(4 k_{1}^{2}-4\right)-n^{2}\right]}{4(2-n)^{2}\left(k_{1} t+k_{2}\right)^{2}} \\
& +\frac{\omega k_{3}^{2}}{2\left(k_{1} t+k_{2}\right)^{2(n+2) /(2-n)}} \\
& -\frac{\rho_{0}}{\left(k_{1} t+k_{2}\right)^{\left(1+w^{(m)}\right)(n+2) /(2-n)}} .
\end{aligned}
$$


From (22) and (30), we get the skewness parameter

$$
\begin{array}{rl}
\gamma=4 & m n(n+2) k_{1}^{2} \\
\times & {\left[\left(12\left(k_{1}^{2}-1\right)+12 n\left(2 k_{1}^{2}+1\right)-3 n^{2}\right)\right.} \\
& +6 \omega(2-n)^{2} k_{3}^{2}\left(k_{1} t+k_{2}\right)^{4 n /(n-2)} \\
& \left.-12 \rho_{0}(2-n)^{2}\left(k_{1} t+k_{2}\right)^{\left((2-3 n)-w^{(m)}(n+2)\right) /(2-n)}\right]^{-1} .
\end{array}
$$

From (23) and (30), we get the skewness parameter

$$
\begin{aligned}
\xi=8 m & (n+2) k_{1}^{2} \\
\times & {\left[12 \rho_{0}(2-n)^{2}\left(k_{1} t+k_{2}\right)^{\left((2-3 n)-w^{(m)}(n+2)\right) /(2-n)}\right.} \\
& -6 \omega(2-n)^{2} k_{3}^{2}\left(k_{1} t+k_{2}\right)^{4 n /(n-2)} \\
& \left.-\left(12\left(k_{1}^{2}-1\right)+12 n\left(2 k_{1}^{2}+1\right)-3 n^{2}\right)\right]^{-1} .
\end{aligned}
$$

From (17) and (26)-(31), we get the EoS parameter of DE

$$
\begin{aligned}
w^{(\mathrm{de})}=- & {\left[k_{5}\left(k_{1} t+k_{2}\right)^{-2}-6 \omega k_{3}^{2}(2-n)^{2}\right.} \\
& \times\left(k_{1} t+k_{2}\right)^{2(n+2) /(n-2)}+12 w^{m} \rho_{0}(2-n)^{2} \\
& \left.\times\left(k_{1} t+k_{2}\right)^{\left(1+w^{(m)}\right)(n+2) /(n-2)}\right] \\
\times & {\left[\left(12\left(k_{1}^{2}-1\right)+12 n\left(2 k_{1}^{2}+1\right)-3 n^{2}\right)\right.} \\
& +6 \omega(2-n)^{2} k_{3}^{2}\left(k_{1} t+k_{2}\right)^{4 n /(n-2)}-12 \rho_{0}(2-n)^{2} \\
& \left.\times\left(k_{1} t+k_{2}\right)^{\left((2-3 n)-w^{(m)}(n+2)\right) /(n-2)}\right]^{-1},
\end{aligned}
$$

where $k_{5}=n^{2}\left[4 k_{1}^{2}(6+m)+3\right]+4 n\left(2 m k_{1}^{2}-3\right)+12\left(k_{1}^{2}-1\right)$.

The density parameters of perfect fluid and DE are as follows:

$$
\begin{aligned}
& \Omega^{(m)}=\frac{\rho^{(m)}}{3 H^{2}} \\
&=\frac{3(2-n)^{2} \rho_{0}}{(n+2)^{2} k_{1}^{2}}\left(k_{1} t+k_{2}\right)^{\left((2-3 n)-w^{(m)}(n+2)\right) /(2-n)}, \\
& n \neq-2,
\end{aligned}
$$

$$
\begin{aligned}
\Omega^{(\mathrm{de})}= & \frac{\rho^{(\mathrm{de})}}{3 H^{2}} \\
= & \frac{\left(12\left(k_{1}^{2}-1\right)+12 n\left(2 k_{1}^{2}+1\right)-3 n^{2}\right)}{4(n+2)^{2} k_{1}^{2}} \\
& +\frac{3 \omega k_{3}^{2}(2-n)^{2}}{2(n+2)^{2} k_{1}^{2}}\left(k_{1} t+k_{2}\right)^{4 n /(n-2)} \\
& -\frac{3(2-n)^{2} \rho_{0}}{(n+2)^{2} k_{1}^{2}}\left(k_{1} t+k_{2}\right)^{\left((2-3 n)-w^{(m)}(n+2)\right) /(2-n)}
\end{aligned}
$$

The overall density parameter $\Omega$ is given by

$$
\begin{aligned}
\Omega= & \Omega^{(m)}+\Omega^{(\mathrm{de})} \\
= & {\left[\frac{\left(12\left(k_{1}^{2}-1\right)+12 n\left(2 k_{1}^{2}+1\right)-3 n^{2}\right)}{8(n-1)^{2} k_{1}^{2}}\right.} \\
& \left.+\frac{3 \omega k_{3}^{2}(2-n)^{2}}{4(n-1)^{2} k_{1}^{2}}\left(k_{1} t+k_{2}\right)^{4 n /(n-2)}\right] A_{m},
\end{aligned}
$$

where $A_{m}$ is the average anisotropy parameter.

The metric (4), in this case, can be written as

$$
\begin{aligned}
d s^{2}= & -d t^{2}+\left(k_{1} t+k_{2}\right)^{2 /(2-n)}\left(d \theta^{2}+d \phi^{2}\right) \\
& +\left(k_{1} t+k_{2}\right)^{2 n /(2-n)}(d \varphi+\theta d \phi)^{2} .
\end{aligned}
$$

Thus the metric (36) together with (28)-(33) constitutes a Bianchi type II perfect fluid dark energy cosmological model in Saez and Ballester [1] theory of gravitation.

Also we observe that the metric (36) together with (28)(33) constitutes a Bianchi type II perfect fluid dark energy cosmological model in general relativity with $k_{3}=0$.

3.2. Bianchi Type VIII $(\delta=-1)$ Cosmological Model. If $\delta=$ $-1,(24)$ can be written as

$$
\frac{\ddot{R}}{R}+C_{1} \frac{\dot{R}^{2}}{R^{2}}+\frac{1}{(n-1) R^{2}}+\frac{R^{2 n-4}}{(n-1)}=0, \quad n \neq 1 .
$$

From (37), for $n=2$ and with suitable substitution, we get

$$
\dot{R}^{2}=C_{2}^{2}-C_{3}^{2} R^{2}
$$

where $C_{1}=(9-16 m) / 3, C_{2}^{2}=3 /(16 m-9)$, and $C_{3}^{2}=3 /(12-$ $16 m), m \neq 3 / 4,9 / 16$.

From (38), we get

$$
R=\left(\frac{C_{2}}{C_{3}}\right) \sin \left(C_{3} t\right) .
$$

From (16) and (39), we get

$$
S=\left[\left(\frac{C_{2}}{C_{3}}\right) \sin \left(C_{3} t\right)\right]^{2} .
$$


From (11), (16), and (39) we get

$$
\begin{aligned}
& \phi^{(r+2) / 2} \\
&=\left[\frac { r + 2 } { 2 } \left(\frac{-C_{4}}{3}\left(\frac{C_{3}}{C_{2}}\right)^{4} \cot \left(C_{3} t\right)\right.\right. \\
&\left.\left.\times\left(\operatorname{cosec}^{2}\left(C_{3} t\right)+2\right)+C_{5}\right)\right], \\
& r \neq-2,
\end{aligned}
$$

where $C_{4}$ and $C_{5}$ are integrating constants.

From (13), we get the energy density of the perfect fluid

$$
\rho^{(m)}=\rho_{0}\left[\left(\frac{C_{2}}{C_{3}}\right) \sin \left(C_{3} t\right)\right]^{-4\left(1+w^{(m)}\right)},
$$

where $\rho_{0}$ is a constant of integration.

From (19) and (39)-(42), we get the dark energy density

$$
\begin{aligned}
\rho^{(\mathrm{de})}=\frac{1}{4 C_{2}^{2}}[ & 20 C_{2}^{2} C_{3}^{2} \cot ^{2}\left(C_{3} t\right)-4 C_{3}^{2} \operatorname{cosec}^{2}\left(C_{3} t\right) \\
& -C_{2}^{2}+2 \omega C_{4}^{2} C_{2}^{2}\left(\left(\frac{C_{2}}{C_{3}}\right) \sin \left(C_{3} t\right)\right)^{-8} \\
& \left.-4 \rho_{0} C_{2}^{2}\left(\left(\frac{C_{2}}{C_{3}}\right) \sin \left(C_{3} t\right)\right)^{-4\left(1+w^{(m)}\right)}\right] .
\end{aligned}
$$

From (22) and (43), we get the skewness parameter

$$
\begin{array}{rl}
\gamma=32 & m C_{2}^{2} C_{3}^{2} \cot ^{2}\left(C_{3} t\right) \\
\times & {\left[60 C_{2}^{2} C_{3}^{2} \cot ^{2}\left(C_{3} t\right)-12 C_{3}^{2} \operatorname{cosec}^{2}\left(C_{3} t\right)-3 C_{2}^{2}\right.} \\
& +6 \omega C_{4}^{2} C_{2}^{2}\left(\left(\frac{C_{2}}{C_{3}}\right) \sin \left(C_{3} t\right)\right)^{-8} \\
& \left.-12 \rho_{0} C_{2}^{2}\left(\left(\frac{C_{2}}{C_{3}}\right) \sin \left(C_{3} t\right)\right)^{-4\left(1+w^{(m)}\right)}\right]^{-1} .
\end{array}
$$

From (23) and (43), we get the skewness parameter

$$
\begin{array}{rl}
\xi=32 & m C_{2}^{2} C_{3}^{2} \cot ^{2}\left(C_{3} t\right) \\
\times & {\left[12 C_{3}^{2} \operatorname{cosec}^{2}\left(C_{3} t\right)+3 C_{2}^{2}-60 C_{2}^{2} C_{3}^{2} \cot ^{2}\left(C_{3} t\right)\right.} \\
& -6 \omega C_{4}^{2} C_{2}^{2}\left(\left(\frac{C_{2}}{C_{3}}\right) \sin \left(C_{3} t\right)\right)^{-8} \\
& \left.+12 \rho_{0} C_{2}^{2}\left(\left(\frac{C_{2}}{C_{3}}\right) \sin \left(C_{3} t\right)\right)^{-4\left(1+w^{(m)}\right)}\right]^{-1} .
\end{array}
$$

From (17) and (39)-(44), we get the EoS parameter of DE

$$
\begin{aligned}
w^{(\mathrm{de})}=- & {\left[3 C_{2}^{2}\left(1-12 C_{3}^{2}\right)+4 C_{2}^{2} C_{3}^{2} \cot ^{2}\left(C_{3} t\right)(12+8 m)\right.} \\
& +12 w^{(m)} \rho_{0} C_{2}^{2}\left(\left(\frac{C_{2}}{C_{3}}\right) \sin \left(C_{3} t\right)\right)^{-4\left(1+w^{(m)}\right)} \\
& \left.-6 \omega C_{4}^{2} C_{2}^{2}\left(\left(\frac{C_{2}}{C_{3}}\right) \sin \left(C_{3} t\right)\right)^{-8}\right] \\
\times & {\left[60 C_{2}^{2} C_{3}^{2} \cot ^{2}\left(C_{3} t\right)-12 C_{3}^{2} \operatorname{cosec}{ }^{2}\left(C_{3} t\right)\right.} \\
& -3 C_{2}^{2}+6 \omega C_{4}^{2} C_{2}^{2}\left(\left(\frac{C_{2}}{C_{3}}\right) \sin \left(C_{3} t\right)\right)^{-8} \\
& \left.-12 \rho_{0} C_{2}^{2}\left(\left(\frac{C_{2}}{C_{3}}\right) \sin \left(C_{3} t\right)\right)^{-4\left(1+w^{(m)}\right)}\right]^{-1} .
\end{aligned}
$$

The density parameters of perfect fluid and DE are as follows:

$$
\begin{aligned}
\Omega^{(m)} & =\frac{\rho^{(m)}}{3 H^{2}} \\
& =\frac{3 \rho_{0}}{16 C_{3}^{2}} \tan ^{2}\left(C_{3} t\right)\left(\left(\frac{C_{2}}{C_{3}}\right) \sin \left(C_{3} t\right)\right)^{-4\left(1+w^{(m)}\right)}, \\
\Omega^{(\mathrm{de})}= & \frac{\rho^{(\mathrm{de})}}{3 H^{2}}=\frac{15}{16}-\frac{3}{16 C_{2}^{2}} \sec ^{2}\left(C_{3} t\right) \\
& -\frac{3 \rho_{0}}{16 C_{3}^{2}} \tan ^{2}\left(C_{3} t\right)\left(\left(\frac{C_{2}}{C_{3}}\right) \sin \left(C_{3} t\right)\right)^{-4\left(1+w^{(m)}\right)} .
\end{aligned}
$$

The overall density parameter $\Omega$ is given by

$$
\Omega=\Omega^{(m)}+\Omega^{(\mathrm{de})}=\left[\frac{15}{2}-\frac{3}{2 C_{2}{ }^{2}} \sec ^{2}\left(C_{3} t\right)\right] A_{m},
$$

where $A_{m}$ is the average anisotropy parameter.

The metric (4), in this case can be written as

$$
\begin{aligned}
d s^{2}= & -d t^{2}+\left(\left(\frac{C_{2}}{C_{3}}\right) \sin \left(C_{3} t\right)\right)^{2}\left(d \theta^{2}+\cosh ^{2} \theta d \phi^{2}\right) \\
& +\left(\left(\frac{C_{2}}{C_{3}}\right) \sin \left(C_{3} t\right)\right)^{4}(d \varphi+\sinh \theta d \phi)^{2} .
\end{aligned}
$$

Thus the metric (49) together with (41)-(46) constitutes a Bianchi type VIII perfect fluid dark energy cosmological model in Saez and Ballester [1] theory of gravitation.

Also we observe that the metric (49) together with (41)(46) constitutes a Bianchi type VIII perfect fluid dark energy cosmological model in general relativity with $C_{4}=0$. 
3.3. Bianchi Type IX $(\delta=1)$ Cosmological Model. If $\delta=1$, (24) can be written as

$$
\frac{\ddot{R}}{R}+C_{1} \frac{\dot{R}^{2}}{R^{2}}-\frac{1}{(n-1) R^{2}}+\frac{R^{2 n-4}}{(n-1)}=0, \quad n \neq 1 .
$$

From (50), for $n=2$ and with suitable substitution, we get

$$
\dot{R}^{2}=C_{2}^{2}-C_{3}^{2} R^{2},
$$

where $C_{1}=(9-16 m) / 3, C_{2}^{2}=3 /(9-16 m)$, and $C_{3}^{2}=3 /(12-$ $16 m), m \neq 3 / 4,9 / 16$.

From (51), we get

$$
R=\left(\frac{C_{2}}{C_{3}}\right) \sin \left(C_{3} t\right) .
$$

From (16) and (52), we get

$$
S=\left[\left(\frac{C_{2}}{C_{3}}\right) \sin \left(C_{3} t\right)\right]^{2} .
$$

From (11), (16), and (52) we get

$$
\begin{aligned}
\phi^{(r+2) / 2} & \\
=\left[\frac{r+2}{2}(\right. & \frac{-C_{4}}{3}\left(\frac{C_{3}}{C_{2}}\right)^{4} \cot \left(C_{3} t\right) \\
& \left.\left.\times\left(\operatorname{cosec}^{2}\left(C_{3} t\right)+2\right)+C_{5}\right)\right], \quad r \neq-2,
\end{aligned}
$$

where $C_{4}$ and $C_{5}$ are integrating constants. One has

From (13), we get the energy density of the perfect fluid

$$
\rho^{(m)}=\rho_{0}\left[\left(\frac{C_{2}}{C_{3}}\right) \sin \left(C_{3} t\right)\right]^{-4\left(1+w^{(m)}\right)},
$$

where $\rho_{0}$ is a constant of integration.

From (19) and (52)-(55), we get the dark energy density

$$
\begin{aligned}
\rho^{(\mathrm{de})}=\frac{1}{4 C_{2}^{2}} & {\left[20 C_{2}^{2} C_{3}^{2} \cot ^{2}\left(C_{3} t\right)+4 C_{3}^{2} \operatorname{cosec}^{2}\left(C_{3} t\right)\right.} \\
& -C_{2}^{2}+2 \omega C_{4}^{2} C_{2}^{2}\left(\left(\frac{C_{2}}{C_{3}}\right) \sin \left(C_{3} t\right)\right)^{-8} \\
& \left.-4 \rho_{0} C_{2}^{2}\left(\left(\frac{C_{2}}{C_{3}}\right) \sin \left(C_{3} t\right)\right)^{-4\left(1+w^{(m)}\right)}\right] .
\end{aligned}
$$

From (22) and (56), we get the skewness parameter

$$
\begin{aligned}
\gamma= & 32 m C_{2}^{2} C_{3}^{2} \cot ^{2}\left(C_{3} t\right) \\
\times & {\left[60 C_{2}^{2} C_{3}^{2} \cot ^{2}\left(C_{3} t\right)+12 C_{3}^{2} \operatorname{cosec}^{2}\left(C_{3} t\right)\right.} \\
& -3 C_{2}^{2}+6 \omega C_{4}^{2} C_{2}^{2}\left(\left(\frac{C_{2}}{C_{3}}\right) \sin \left(C_{3} t\right)\right)^{-8} \\
& \left.-12 \rho_{0} C_{2}^{2}\left(\left(\frac{C_{2}}{C_{3}}\right) \sin \left(C_{3} t\right)\right)^{-4\left(1+w^{(m)}\right)}\right]^{-1} .
\end{aligned}
$$

From (23) and (56), we get the skewness parameter

$$
\begin{aligned}
\xi= & 32 m C_{2}^{2} C_{3}^{2} \cot ^{2}\left(C_{3} t\right) \\
\times & {\left[12 \rho_{0} C_{2}^{2}\left(\left(\frac{C_{2}}{C_{3}}\right) \sin \left(C_{3} t\right)\right)^{-4\left(1+w^{(m)}\right)}\right.} \\
& -12 C_{3}^{2} \operatorname{cosec}^{2}\left(C_{3} t\right)+3 C_{2}^{2}-60 C_{2}^{2} C_{3}^{2} \cot ^{2}\left(C_{3} t\right) \\
& \left.-6 \omega C_{4}^{2} C_{2}^{2}\left(\left(\frac{C_{2}}{C_{3}}\right) \sin \left(C_{3} t\right)\right)^{-8}\right]^{-1} .
\end{aligned}
$$

From (17) and (52)-(57), we get the EoS parameter of DE

$$
\begin{aligned}
w^{(\mathrm{de})}=- & {\left[3 C_{2}^{2}\left(1-12 C_{3}^{2}\right)+4 C_{2}^{2} C_{3}^{2} \cot ^{2}\left(C_{3} t\right)(12+8 m)\right.} \\
& +12 w^{(m)} \rho_{0} C_{2}^{2}\left(\left(\frac{C_{2}}{C_{3}}\right) \sin \left(C_{3} t\right)\right)^{-4\left(1+w^{(m)}\right)} \\
& \left.-6 \omega C_{4}^{2} C_{2}^{2}\left(\left(\frac{C_{2}}{C_{3}}\right) \sin \left(C_{3} t\right)\right)^{-8}\right] \\
\times & {\left[12 C_{3}^{2} \operatorname{cosec}^{2}\left(C_{3} t\right)\right.} \\
& -12 \rho_{0} C_{2}^{2}\left(\left(\frac{C_{2}}{C_{3}}\right) \sin \left(C_{3} t\right)\right)^{-4\left(1+w^{(m)}\right)} \\
& -3 C_{2}^{2}+60 C_{2}^{2} C_{3}^{2} \cot ^{2}\left(C_{3} t\right) \\
& \left.+6 \omega C_{4}^{2} C_{2}^{2}\left(\left(\frac{C_{2}}{C_{3}}\right) \sin \left(C_{3} t\right)\right)^{-8}\right]^{-1} .
\end{aligned}
$$

The density parameters of perfect fluid and DE are as follows:

$$
\begin{aligned}
\Omega^{(m)}= & \frac{\rho^{(m)}}{3 H^{2}} \\
= & \frac{3 \rho_{0}}{16 C_{3}^{2}} \tan ^{2}\left(C_{3} t\right)\left(\left(\frac{C_{2}}{C_{3}}\right) \sin \left(C_{3} t\right)\right)^{-4\left(1+w^{(m)}\right)}, \\
\Omega^{(\mathrm{de})}= & \frac{\rho^{(\mathrm{de})}}{3 H^{2}}=\frac{15}{16}+\frac{3}{16 C_{2}^{2}} \sec ^{2}\left(C_{3} t\right) \\
& -\frac{3 \rho_{0}}{16 C_{3}^{2}} \tan ^{2}\left(C_{3} t\right)\left(\left(\frac{C_{2}}{C_{3}}\right) \sin \left(C_{3} t\right)\right)^{-4\left(1+w^{(m)}\right)} .
\end{aligned}
$$

The overall density parameter $\Omega$ is given by

$$
\Omega=\Omega^{(m)}+\Omega^{(\mathrm{de})}=\left[\frac{15}{2}+\frac{3}{2 C_{2}^{2}} \sec ^{2}\left(C_{3} t\right)\right] A_{m}
$$

where $A_{m}$ is the average anisotropy parameter. 
The metric (4), in this case, can be written as

$$
\begin{aligned}
d s^{2}= & -d t^{2}+\left(\left(\frac{C_{2}}{C_{3}}\right) \sin \left(C_{3} t\right)\right)^{2}\left(d \theta^{2}+\sin \theta d \phi^{2}\right) \\
& +\left(\left(\frac{C_{2}}{C_{3}}\right) \sin \left(C_{3} t\right)\right)^{4}(d \varphi+\cos \theta d \phi)^{2} .
\end{aligned}
$$

Thus the metric (62) together with (54)-(59) constitutes a Bianchi type IX perfect fluid dark energy cosmological model in Saez and Ballester [1] theory of gravitation.

Also we observe that the metric (62) together with (54)(59) constitutes a Bianchi type IX perfect fluid dark energy cosmological model in general relativity with $C_{4}=0$.

3.4. Isotropic Cosmological Models. To get isotropic cosmological models, let us assign the value unity to $n$ in (16) and then we get

$$
R=S \text {, }
$$

where $R$ and $S$ are the metric potentials.

Using (63), the field equations (17)-(20) can be written as

$$
\begin{aligned}
& 2 \frac{\ddot{S}}{S} \frac{\dot{S}^{2}}{S^{2}}+\frac{1}{4 S^{2}}-\frac{\omega}{2} \phi^{r} \dot{\phi}^{2} \\
&=-w^{(m)} \rho^{(m)}-\left(w^{(\mathrm{de})}+\gamma\right) \rho^{(\mathrm{de})}, \\
& 2 \frac{\ddot{S}}{S} \frac{\dot{S}^{2}}{S^{2}}+\frac{\delta}{S^{2}}-\frac{3}{4 S^{2}}-\frac{\omega}{2} \phi^{r} \dot{\phi}^{2} \\
&=-w^{(m)} \rho^{(m)}-\left(w^{(\mathrm{de})}+\xi\right) \rho^{(\mathrm{de})}, \\
& 3 \frac{\dot{S}^{2}}{S^{2}}+ \frac{\delta}{S^{2}}-\frac{1}{4 S^{2}}+\frac{\omega}{2} \phi^{r} \dot{\phi}^{2}=\rho^{(m)}+\rho^{(\mathrm{de})}, \\
& \dot{\phi}+3 \frac{\dot{\phi} \dot{S}}{S}+\frac{r}{2 \phi} \dot{\phi}^{2}=0 .
\end{aligned}
$$

From (64) and (65), we get

$$
3 m \dot{S}^{2}+(\delta-1)=0 .
$$

3.5. Bianchi Type II and VIII Cosmological Models in Isotropic Form. From (68), we get

$$
R=S=\left(C_{6} t+C_{7}\right),
$$

where $C_{6}=((1-\delta) / 3 m)^{1 / 2}$ and $C_{7}$ are constants.

From (67) and (69) we get

$$
\phi^{(r+2) / 2}=\frac{r+2}{2}\left(C_{9}-\frac{C_{8}}{2\left(C_{6} t+C_{7}\right)^{2}}\right), \quad r \neq-2,
$$

where $C_{8}$ and $C_{9}$ are integrating constants.

From (13), we get the energy density of the perfect fluid

$$
\rho^{(m)}=\rho_{0}\left(C_{6} t+C_{7}\right)^{-3\left(1+w^{(m)}\right)},
$$

where $\rho_{0}$ is a constant of integration.
From (66), (69), and (71), we get the dark energy density

$$
\begin{aligned}
\rho^{(\mathrm{de})}= & 3 C_{6}^{2}+\frac{(4 \delta-1)}{4}\left(C_{6} t+C_{7}\right)^{-2} \\
& +\frac{\omega C_{8}^{2}}{2}\left(C_{6} t+C_{7}\right)^{-6}-\rho_{0}\left(C_{6} t+C_{7}\right)^{-3\left(1+w^{(m)}\right)} .
\end{aligned}
$$

From (22) and (72), we get the skewness parameter

$$
\begin{aligned}
\gamma=-m C_{6}{ }^{2} & {\left[3 C_{6}{ }^{2}+\frac{(4 \delta-1)}{4}\left(C_{6} t+C_{7}\right)^{-2}\right.} \\
& +\frac{\omega C_{8}^{2}}{2}\left(C_{6} t+C_{7}\right)^{-6} \\
& \left.-\rho_{0}\left(C_{6} t+C_{7}\right)^{-3\left(1+w^{(m)}\right)}\right]^{-1} .
\end{aligned}
$$

From (23) and (72), we get the skewness parameter

$$
\begin{aligned}
\xi=2 m C_{6}^{2} & {\left[3 C_{6}^{2}+\frac{(4 \delta-1)}{4}\left(C_{6} t+C_{7}\right)^{-2}\right.} \\
& +\frac{\omega C_{8}^{2}}{2}\left(C_{6} t+C_{7}\right)^{-6} \\
& \left.-\rho_{0}\left(C_{6} t+C_{7}\right)^{-3\left(1+w^{(m)}\right)}\right]^{-1}
\end{aligned}
$$

From (64) and (69)-(73), we get the EoS parameter of DE

$$
\begin{aligned}
w^{(\mathrm{de})}=- & {\left[(1-m) C_{6}^{2}+\frac{1}{4}\left(C_{6} t+C_{7}\right)^{-2}\right.} \\
& -\frac{\omega C_{8}^{2}}{2}\left(C_{6} t+C_{7}\right)^{-6} \\
& \left.+\rho_{0} w^{(m)}\left(C_{6} t+C_{7}\right)^{-3\left(1+w^{(m)}\right)}\right] \\
\times & {\left[3 C_{6}^{2}+\frac{(4 \delta-1)}{4}\left(C_{6} t+C_{7}\right)^{-2}\right.} \\
& +\frac{\omega C_{8}^{2}}{2}\left(C_{6} t+C_{7}\right)^{-6} \\
& \left.-\rho_{0}\left(C_{6} t+C_{7}\right)^{-3\left(1+w^{(m)}\right)}\right]^{-1} .
\end{aligned}
$$

The density parameters of perfect fluid and DE are as follows:

$$
\begin{aligned}
\Omega^{(m)} & =\frac{\rho^{(m)}}{3 H^{2}} \\
& =\frac{\rho_{0}}{3 C_{6}^{2}}\left(C_{6} t+C_{7}\right)^{-\left(1+3 w^{(m)}\right)}, \\
\Omega^{(\mathrm{de})}= & \frac{\rho^{(\mathrm{de})}}{3 H^{2}} \\
= & \left(C_{6} t+C_{7}\right)^{2}-\frac{5}{12 C_{6}^{2}}+\frac{\omega C_{8}^{2}}{6 C_{6}^{2}}\left(C_{6} t+C_{7}\right)^{-4} \\
& -\frac{\rho_{0}}{3 C_{6}^{2}}\left(C_{6} t+C_{7}\right)^{-\left(1+3 w^{(m)}\right)} .
\end{aligned}
$$


The overall density parameter $\Omega$ is given by

$$
\begin{aligned}
\Omega= & \Omega^{(m)}+\Omega^{(\mathrm{de})} \\
= & \left(C_{6} t+C_{7}\right)^{2}-\frac{5}{12 C_{6}^{2}} \\
& +\frac{\omega C_{8}^{2}}{6 C_{6}^{2}}\left(C_{6} t+C_{7}\right)^{-4} .
\end{aligned}
$$

The metric (4), in this case, can be written as

$$
\begin{aligned}
d s^{2}= & -d t^{2}+\left(C_{6} t+C_{7}\right)^{2}\left[d \theta^{2}+f(\theta) d \phi^{2}\right] \\
& +\left(C_{6} t+C_{7}\right)^{2}[d \varphi+h(\theta) d \phi]^{2} .
\end{aligned}
$$

The metric (78) together with (70) to (75) constitutes Bianchi type II and VIII perfect fluid dark energy cosmological models in isotropic form of Saez and Ballester [1] theory of gravitation, respectively, with $\delta=0, f(\theta)=1$, and $h(\theta)=\theta$ and $\delta=0, f(\theta)=\cosh \theta$ and $h(\theta)=\sinh \theta$.

Also we can observe that the metric (78) together with (70) to (75) constitutes Bianchi type II and VIII perfect fluid dark energy cosmological models in isotropic form of general relativity with $C_{8}=0$ respectively for $\delta=0, f(\theta)=$ 1 , and $h(\theta)=\theta$ and $\delta=-1, f(\theta)=\cosh \theta$ and $h(\theta)=\sinh \theta$.

3.6. Bianchi Type IX $(\delta=1)$ Cosmological Model in Isotropic Form. From (68), if $\delta=1$, we get

$$
R=S=\text { cons } \tan t\left(\text { say } C_{10}\right)
$$

From (67) and (79), we get

$$
\phi^{(r+2) / 2}=\left[\frac{r+2}{2}\left(C_{11} t+C_{12}\right)\right] .
$$

The metric (4), in this case, can be written as

$$
\begin{aligned}
d s^{2}= & -d t^{2}+C_{10}^{2}\left(d \theta^{2}+\sin \theta d \phi^{2}\right) \\
& +C_{10}^{2}(d \varphi+\cos \theta d \phi)^{2}
\end{aligned}
$$

The metric (81) together with (80) constitutes flat cosmological model in Saez and Ballester [1] theory of gravitation.

\section{Some Other Important Features of the Models}

4.1. Bianchi Type II Anisotropic Cosmological Model $(\delta=0)$. The spatial volume for the model (36) is

$$
V=a^{3}=\left(k_{1} t+k_{2}\right)^{(n+2) /(2-n)} .
$$

The expression for expansion scalar $\theta$ calculated for the flow vector $u^{i}$ is given by

$$
\theta=3 H=\left(\frac{n+2}{2-n}\right) \frac{k_{1}}{\left(k_{1} t+k_{2}\right)}
$$

and the shear $\sigma$ is given by

$$
\sigma^{2}=\frac{1}{2}\left(\sum_{i=1}^{3} H_{i}^{2}-\frac{1}{3} \theta^{2}\right)=\left(\frac{n-1}{2-n}\right)^{2} \frac{k_{1}^{2}}{3\left(k_{1} t+k_{2}\right)^{2}}
$$

The deceleration parameter $q$ is given by

$$
q=\frac{d}{d t}\left(\frac{1}{H}\right)-1=\frac{4(1-n)}{(n+2)}, \quad n \neq 1,2 \&-2 .
$$

The recent observations of SN Ia reveal that the present universe is accelerating and the value of deceleration parameter lies somewhere in the range $-1<q<0$. From (85), we can see that for $n>2, q$ is negative, which may be attributed to the current accelerated expansion of the universe.

The components of Hubble parameter $H_{x}, H_{y}$, and $H_{z}$ are given by

$$
\begin{gathered}
H_{x}=H_{y}=\frac{\dot{R}}{R}=\left(\frac{1}{2-n}\right) \frac{k_{1}}{\left(k_{1} t+k_{2}\right)}, \\
H_{z}=\frac{\dot{S}}{S}=\left(\frac{n}{2-n}\right) \frac{k_{1}}{\left(k_{1} t+k_{2}\right)} .
\end{gathered}
$$

Therefore the generalized mean Hubble parameter $(H)$ is

$$
H=\frac{1}{3}\left(H_{x}+H_{y}+H_{z}\right)=\left(\frac{n+2}{2-n}\right) \frac{k_{1}}{3\left(k_{1} t+k_{2}\right)} .
$$

The average anisotropy parameter is defined by

$$
A_{m}=\frac{1}{3} \sum_{i=1}^{3}\left(\frac{\Delta H_{i}}{H}\right)^{2}=\frac{2(n-1)^{2}}{(n+2)^{2}},
$$

where $\Delta H_{i}=H_{i}-H(i=1,2,3)$.

A cosmological diagnostic pair $\{r, s\}$ called state finder as proposed by Sahni et al. [39] is given by

$$
\begin{aligned}
& r=\frac{\dddot{a}}{a H^{3}}=\left(1+\frac{3(n-2)}{(n+2)}\right)\left(1+\frac{6(n-2)}{(n+2)}\right), \\
s= & \frac{r-1}{3(q-(1 / 2))} \\
= & \frac{1-(1+3(n-2) /(n+2))(1+6(n-2) /(n+2))}{9((n-2) /(n+2)+1 / 2)} .
\end{aligned}
$$


The dynamics of state finder $\{r, s\}$ depends on $n$. It follows that in the derived model, one can choose the pair of state finder, which can successfully differentiate between a wide variety of DE models including cosmological constant, quintessence, phantom, quintom, the Chaplygin gas, braneworld models, and interacting DE models. For example if we put $n=2$, the state finder pair will be $\{1,0\}$ which yields the $\Lambda C D M$ (cosmological constant cold dark matter) model.

4.2. Bianchi Type VIII $(\delta=-1)$ and IX $(\delta=1)$ Anisotropic Cosmological Models. Thespatial volume for both the models (49) and (62) is

$$
V=a^{3}=\left(\left(\frac{C_{2}}{C_{3}}\right) \sin \left(C_{3} t\right)\right)^{4} f(\theta),
$$

where $f(\theta)=\cosh \theta$ and $\sin \theta$ for Bianchi type VIII and IX, respectively.

The expression for expansion scalar $\theta$ and the shear $\sigma$ for the models (49) and (62) are given by

$$
\begin{gathered}
\theta=3 H=4 C_{3} \cot \left(C_{3} t\right), \\
\sigma^{2}=\frac{1}{2}\left(\sum_{i=1}^{3} H_{i}^{2}-\frac{1}{3} \theta^{2}\right)=\frac{C_{3}^{2} \cot ^{2}\left(C_{3} t\right)}{3} .
\end{gathered}
$$

The deceleration parameter $q$ for the models (49) and (62) is given by

$$
q=\frac{d}{d t}\left(\frac{1}{H}\right)-1=\frac{3}{4} \sec ^{2}\left(C_{3} t\right)-1 .
$$

From (92), we can observe that the deceleration parameter $q$ is always negative and hence they represent accelerating universes.

The components of the Hubble parameter $H_{x}, H_{y}$, and $H_{z}$ for the models (49) and (62) are given by

$$
\begin{gathered}
H_{x}=H_{y}=\frac{\dot{R}}{R}=C_{3} \cot \left(C_{3} t\right), \\
H_{z}=\frac{\dot{S}}{S}=2 C_{3} \cot \left(C_{3} t\right) .
\end{gathered}
$$

Therefore the generalized mean Hubble parameter $(H)$ is

$$
H=\frac{1}{3}\left(H_{x}+H_{y}+H_{z}\right)=\frac{4}{3} C_{3} \cot \left(C_{3} t\right)
$$

The average anisotropy parameters for the models (49) and (62) are defined by

$$
A_{m}=\frac{1}{3} \sum_{i=1}^{3}\left(\frac{\Delta H_{i}}{H}\right)^{2}=\frac{1}{8}
$$

where $\Delta H_{i}=H_{i}-H(i=1,2,3)$.

A cosmological diagnostic pair $\{r, s\}$ called state finder as proposed by Sahni et al. [39] is given by

$$
\begin{gathered}
r=\frac{\dddot{a}}{a H^{3}}=\frac{-1}{8}\left(1+9 \tan ^{2} C_{3} t\right), \\
s=\frac{r-1}{3(q-(1 / 2))}=\frac{-1}{2} \frac{\sec ^{2} C_{3} t}{\left(\tan ^{2} C_{3} t-1\right)} .
\end{gathered}
$$

4.3. Bianchi Type II $(\delta=0)$ and VIII $(\delta=-1)$ Cosmological Models in Isotropic Form. Thespatial volume for the above models is given by

$$
V\left(C_{6} t+C_{7}\right)^{3}
$$

The expression for expansion scalar $\theta$ calculated for the flow vector $u^{i}$ is given by

$$
\theta=3 H=\frac{3 C_{6}}{\left(C_{6} t+C_{7}\right)}
$$

and the shear $\sigma$ is given by

$$
\sigma^{2}=\frac{1}{2}\left(\sum_{i=1}^{3} H_{i}^{2}-\frac{1}{3} \theta^{2}\right)=\frac{-3 C_{6}^{2}}{\left(C_{6} t+C_{7}\right)^{2}} .
$$

The deceleration parameter $q$ is given by

$$
q=\frac{d}{d t}\left(\frac{1}{H}\right)-1=0
$$

The components of Hubble parameter $H_{x}, H_{y}$, and $H_{z}$ are given by

$$
H_{x}=H_{y}=H_{z}=\frac{C_{6}}{\left(C_{6} t+C_{7}\right)} .
$$

Therefore the generalized mean Hubble parameter $(H)$ is

$$
H=\frac{1}{3}\left(H_{x}+H_{y}+H_{z}\right)=\frac{C_{6}}{\left(C_{6} t+C_{7}\right)} .
$$

The average anisotropy parameter is

$$
A_{m}=\frac{1}{3} \sum_{i=1}^{3}\left(\frac{\Delta H_{i}}{H}\right)^{2}=0
$$

where $\Delta H_{i}=H_{i}-H(i=1,2,3)$.

\section{Conclusions}

In this paper, we have presented spatially homogeneous anisotropic Bianchi type II, VIII and IX as well as isotropic space times filled with perfect fluid and DE possessing dynamical energy density in Saez-Ballester [1] scalar-tensor theory of gravitation and general relativity. Studying the interaction between the ordinary matter and DE will open up the possibility of detecting DE. For Bianchi type II cosmological model, we observe that at $t=-k_{2} / k_{1}$, the spatial volume vanishes while all other parameters diverge for $1<n<2$. Thus the derived model starts expanding with big bang singularity at $t=-k_{2} / k_{1}$ which can be shifted to $t=0$ by choosing $k_{2}=0$. The model has point type singularity at $t=$ $-k_{2} / k_{1}$ when $0<n<2(n \neq 1)$. Also the model has cigar type singularity at $t=-k_{2} / k_{1}$ when $n<0$ and also for $n>2$. For Bianchi type VIII and IX cosmological models, we observe that the spatial volume increases as time increases and also the models have no initial singularity at $t=0$. The expansion scalar $\theta$ and the shear scalar $\sigma$ decrease as time increases. In 
the derived models, the EoS parameter of DE $w^{(\mathrm{de})}$ is obtained as time varies and it is evolving with negative sign which may be attributed to the current accelerated expansion of universe.

For the isotropic cosmological models (78), we observe that the spatial volume increases as time increases and also the models have no initial singularity at $t=-C_{7} / C_{6}$. Thus the derived models start expanding with big bang singularity at $t=-C_{7} / C_{6}$ which can be shifted to $t=0$ by choosing $C_{7}=0$. The expansion scalar $\theta$, shear scalar $\sigma$, and the Hubble parameter $H$ decrease as time increases. Since $q=0$, the expansion of the isotropic universes proceeds at a constant rate. It may be noted that Bianchi type II, VIII, and IX dark energy cosmological models filled with perfect fluid represent the cosmos in its early stage of evolution and isotropic models represent the present universe.

\section{Acknowledgment}

The second author (K. V. S. Sireesha) is grateful to the Department of Science and Technology (DST), New Delhi, India, for providing INSPIRE fellowship.

\section{References}

[1] D. Sáez and V. J. Ballester, "A simple coupling with cosmological implications," Physics Letters A, vol. 113, no. 9, pp. 467-470, 1986.

[2] V. U. M. Rao, T. Vinutha, and M. Vijaya Santhi, "An exact Bianchi type-V cosmological model in Saez-Ballester theory of gravitation," Astrophysics and Space Science, vol. 312, no. 3-4, pp. 189-191, 2007.

[3] V. U. M. Rao, M. Vijaya Santhi, and T. Vinutha, "Exact Bianchi type II, VIII and IX string cosmological models in SaezBallester theory of gravitation," Astrophysics and Space Science, vol. 314, no. 1-3, pp. 73-77, 2008.

[4] V. U. M. Rao, M. Vijaya Santhi, and T. Vinutha, "Exact Bianchi type-II, VIII and IX perfect fluid cosmological models in SaezBallester theory of gravitation," Astrophysics and Space Science, vol. 317, no. 1-2, pp. 27-30, 2008.

[5] R. L. Naidu, B. Satyanarayana, and D. R. K. Reddy, "LRS Bianchi type-II Universe with cosmic strings and bulk viscosity in a scalar tensor theory of gravitation," Astrophysics and Space Science, vol. 338, no. 2, pp. 351-354, 2012.

[6] R. L. Naidu, B. Satyanarayana, and D. R. K. Reddy, "LRS Bianchi type-II dark energy model in a scalar-tensor theory of gravitation," Astrophysics and Space Science, vol. 338, no. 2, pp. 333-336, 2012.

[7] R. L. Naidu, B. Satyanarayana, and D. R. K. Reddy, "Bianchi type-III dark energy model in a Saez-Ballester scalar-tensor theory," International Journal of Theoretical Physics, vol. 51, no. 9, pp. 2857-2862, 2012.

[8] R. L. Naidu, B. Satyanarayana, and D. R. K. Reddy, "Bianchi type-V dark energy model in a scalar-tensor theory of gravitation," International Journal of Theoretical Physics, vol. 51, no. 7, pp. 1997-2002, 2012.

[9] S. Perlmutter, S. Gabi, G. Goldhaber et al., "Measurements of the cosmological parameters $\Omega$ and $\wedge$ from the first seven supernovae at $z \geq 0.35$," The Astrophysical Journal, vol. 483, no. 2, p. 565, 1997.
[10] S. Perlmutter, G. Aldering, M. Della Valle et al., "Discovery of a supernova explosion at half the age of the Universe," Nature, vol. 391, pp. 51-54, 1998.

[11] S. Perlmutter, G. Aldering, G. Goldhaber et al., "Measurements of $\Omega$ and $\wedge$ from 42 high-redshift supernovae," The Astrophysical Journal, vol. 517, no. 2, p. 565, 1999.

[12] A. G. Riess, A. V. Filippenko, P. Challis et al., "Observational evidence from supernovae for an accelerating universe and a cosmological constant," The Astronomical Journal, vol. 116, no. 3, p. 1009, 1998.

[13] A. G. Riess, L.-G. Strolger, J. Tonry et al., “Type Ia Supernova Discoveries at $z>1$ from the Hubble Space Telescope: evidence for past deceleration and constraints on dark energy evolution," The Astronomical Journal, vol. 607, no. 2, p. 665, 2004.

[14] C. Fedeli, L. Moscardini, and M. Bartelmann, "Observing the clustering properties of galaxy clusters in dynamical darkenergy cosmologies," Astronomy \& Astrophysics, vol. 500, no. 2, pp. 667-679, 2009.

[15] R. R. Caldwell and M. Doran, "Cosmic microwave background and supernova constraints on quintessence: concordance regions and target models," Physical Review D, vol. 69, Article ID 103517, 6 pages, 2004.

[16] Z.-Y. Huang, B. Wang, E. Abdalla, and R.-K. Sul, "Holographic explanation of wide-angle power correlation suppression in the cosmic microwave background radiation," Journal of Cosmology and Astroparticle Physics, vol. 2006, article 013, 2006.

[17] S. F. Daniel, R. R. Caldwell, A. Cooray, and A. Melchiorri, "Large scale structure as a probe of gravitational slip," Physical Review D, vol. 77, no. 10, Article ID 103513, 12 pages, 2008.

[18] R. R. Caldwell, W. Komp, L. Parker, and D. A. T. Vanzella, "Sudden gravitational transition," Physical Review D, vol. 73, no. 2, Article ID 023513, 8 pages, 2006.

[19] P. J. E. Peebles and B. Ratra, "The cosmological constant and dark energy," Reviews of Modern Physics, vol. 75, no. 2, pp. 559606, 2003.

[20] G. Hinshaw, J. L. Weiland, N. Odegard et al., "Five-year Wilkinson Microwave Anisotropy Probe observations: data processing, sky maps, and basic results," The Astrophysical Journal, vol. 180, no. 2 , p. $225,2009$.

[21] E. J. Copeland, M. Sami, and S. Tsujikava, "Dynamics of dark energy," International Journal of Modern Physics D, vol. 15, no. 11, p. 1753, 2006.

[22] P. J. Steinhardt, L. M. Wang, and I. Zlatev, "Cosmological tracking solutions," Physical Review D, vol. 59, Article ID 123504, 13 pages, 1999.

[23] R. R. Caldwell, "A phantom menace? Cosmological consequences of a dark energy component with super-negative equation of state," Physics Letters B, vol. 545, no. 1-2, pp. 23-29, 2002.

[24] P. Astier, J. Guy, N. Regnault et al., “The supernova legacy survey: measurement of $\Omega_{\mathrm{M}}, \Omega_{\wedge}$ and $w$ from the first year data set," Astronomy \& Astrophysics, vol. 447, no. 1, pp. 31-48, 2006.

[25] D. J. Eisentein, I. Zehavi, D. W. Hogg et al., "Detection of the baryon acoustic peak in the large-scale correlation function of SDSS luminous red galaxies," The Astrophysical Journal, vol. 633, no. 2, p. 560, 2005.

[26] C. J. MacTavish, P. A. R. Ade, J. J. Bock et al., "Cosmological parameters from the 2003 flight of BOOMERANG," The Astrophysical Journal, vol. 647, no. 2, p. 799, 2006.

[27] E. Komatsu, J. Dunkley, M. R. Nolta et al., "Five-year Wilkinson Microwave Anisotropy Probe observations: cosmological interpretation," The Astrophysical Journal, vol. 180, no. 2, p. 330, 2009. 
[28] M. Tegmark, M. R. Blanton, M. A. Strauss et al., "The threedimensional power spectrum of galaxies from the sloan digital sky survey," The Astrophysical Journal, vol. 606, no. 2, p. 702, 2004.

[29] S. Kumar and C. P. Singh, "Anisotropic Bianchi type-I models with constant deceleration parameter in general relativity," Astrophysics and Space Science, vol. 312, no. 1-2, pp. 57-62, 2007.

[30] Ö Akarsu and C. B. Kilinc, "LRS Bianchi type I models with anisotropic dark energy and constant deceleration parameter," General Relativity and Gravitation, vol. 42, no. 1, pp. 119-140, 2010.

[31] Ö Akarsu and C. B. Kilinc, "Bianchi type III models with anisotropic dark energy," General Relativity and Gravitation, vol. 42, no. 4, pp. 763-775, 2010.

[32] Ö Akarsu and C. B. Kilinc, "de Sitter expansion with anisotropic fluid in Bianchi type-I space-time," Astrophysics and Space Science, vol. 326, no. 2, pp. 315-322, 2010.

[33] A. K. Yadav, F. Rahaman, and S. Ray, "Dark energy models with variable equation of state parameter," International Journal of Theoretical Physics, vol. 50, no. 3, pp. 871-881, 2010.

[34] A. K. Yadav and L. Yadav, "Bianchi type III anisotropic dark energy models with constant deceleration parameter," International Journal of Theoretical Physics, vol. 50, no. 1, pp. 218-227, 2010.

[35] A. Pradhan, H. Amirhashchi, and B. Saha, "Bianchi typeI anisotropic dark energy model with constant deceleration parameter," International Journal of Theoretical Physics, vol. 50, no. 9, pp. 2923-2938, 2011.

[36] V. U. M. Rao, G. Sreedevi Kumari, and D. Neelima, "A dark energy model in a scalar tensor theory of gravitation," Astrophysics and Space Science, vol. 337, no. 1, pp. 499-501, 2012.

[37] V. U. M. Rao, M. Vijaya Santhi, T. Vinutha, and G. Sreedevi Kumari, "LRS Bianchi type-I dark energy cosmological model in Brans-Dicke theory of gravitation," International Journal of Theoretical Physics, vol. 51, no. 10, pp. 3303-3310, 2012.

[38] D. R. K. Reddy, B. Satyanarayana, and R. L. Naidu, "Five dimensional dark energy model in a scalar-tensor theory of gravitation," Astrophysics and Space Science, vol. 339, no. 2, pp. 401-404, 2012.

[39] V. Sahni, T. D. Saini, A. A. Starobinsky, and U. Alam, "Statefinder-a new geometrical diagnostic of dark energy," Journal of Experimental and Theoretical Physics Letters, vol. 77, no. 5, pp. 201-206, 2003. 

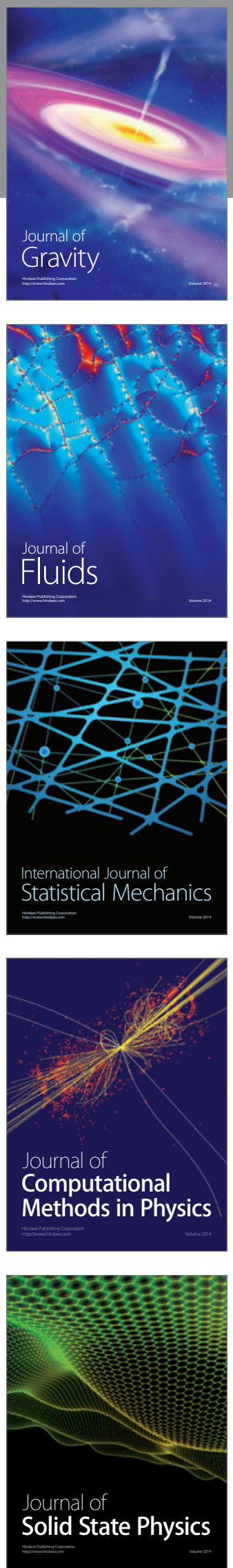

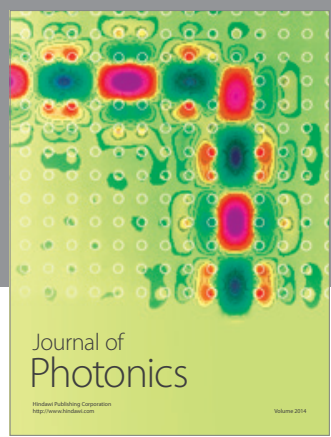

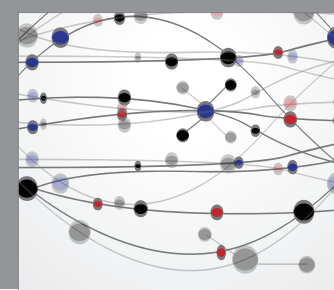

The Scientific World Journal

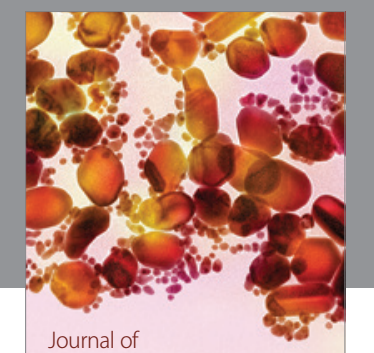

Soft Matter
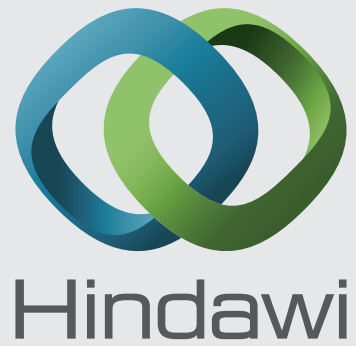

Submit your manuscripts at

http://www.hindawi.com
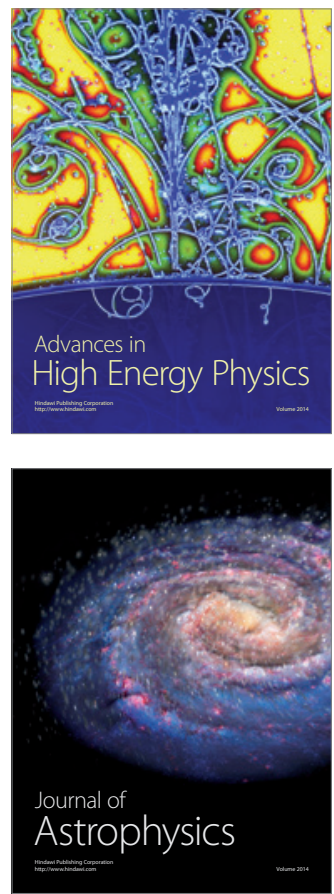
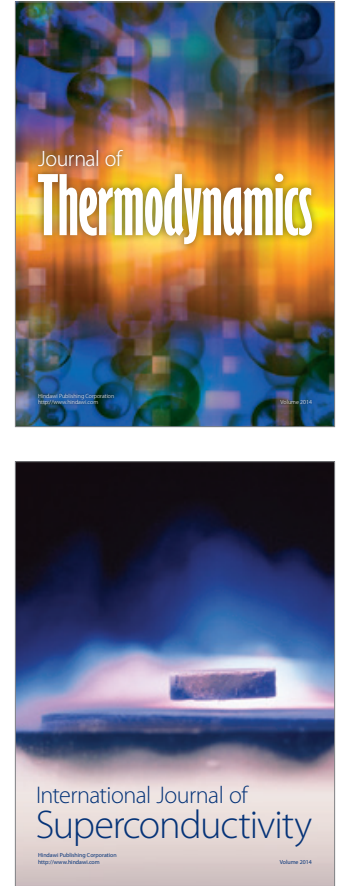
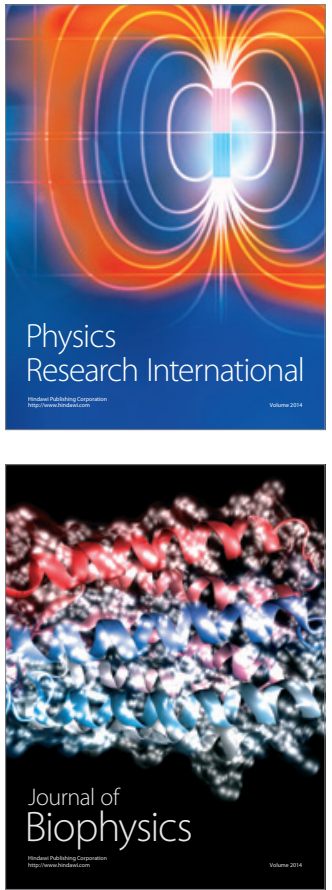
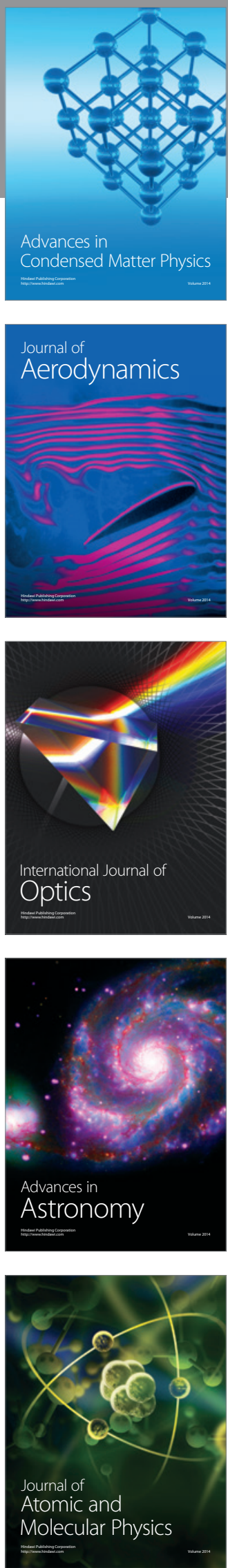